\title{
Off-label use of recombinant activated coagulation factor VII for bleeding may raise the risk of arterial thrombosis
}

\author{
Laura Ferrari · Gian Marco Podda
}

Received: 23 March 2011 / Accepted: 12 April 2011/Published online: 4 May 2011

(C) SIMI 2011

\section{Background}

Recombinant activated coagulation factor VII (rFVIIa), structurally nearly identical to human plasma-derived coagulation factor VIIa, is thought to promote haemostasis by activating coagulation cascade, and converting prothrombin to thrombin. Initially, recombinant factor VIIa was approved for the treatment of bleeding in patients with haemophilia $\mathrm{A}$ or $\mathrm{B}$ who have inhibiting antibodies to coagulation factor VIII or IX. Later, indications were broadened to include the treatment of bleeding events, and the prevention of bleeding episodes related to surgical or invasive procedures, in patients with congenital or acquired haemophilia, factor VII deficiency, or Glanzmann's thrombasthenia. However, in recent years, rFVIIa has been used, on an off-label basis, to act as a pro-haemostatic agent in patients with a pre-existent normal coagulation system who experienced massive or uncontrolled bleeding. In the past decade, the use of rFVIIa for the approved haemophilia indications remained stable over time, whereas the off-label prescriptions increased: according to the recent surveys, more than $90 \%$ of rFVIIa prescriptions are for off-label indications [1,2]. Given the physiology of activated factor VII, a potential adverse event of concern is the risk of thromboembolic complications.

On behalf of GrAM.

L. Ferrari · G. M. Podda $(\square)$

Medicina III, Ospedale San Paolo, Università degli Studi di Milano, Milan, Italy

e-mail: gmpodda@gmail.com

\section{Summary}

Data from 35 randomized clinical trials of off-label indications for rFVIIa versus placebo (26 studies involving patients and nine studies involving healthy volunteers) were analysed to determine the frequency of thromboembolic events [3]. Most of the clinical trials were Novo Nordisk-sponsored studies (29 vs. 6 investigator-initiated trials). The data were pooled and the rates of thromboembolic events were calculated in rFVIIa and placebo groups. Odds ratios for thromboembolic events, adjusted for age, type of bleeding, type of adverse event and dose of rFVIIa, were calculated with the use of logistic regression. A total of 4,468 subjects (4,119 patients and 349 healthy volunteers) were included. Most of them were patients with spontaneous central nervous system bleeding (31.3\%), followed by bleeding due to advanced liver disease $(27.8 \%)$, trauma $(18.7 \%)$ cardiac surgery $(6 \%)$ or spinal surgery $(1.1 \%)$ and bleeding from other causes $(5.1 \%)$. Healthy volunteers were 349 ( $7.8 \%$ of the subjects).

When the results were adjusted for age and type of bleeding, there was no significant difference $(p=0.16)$ in the rate of thromboembolic events between patients who received $\mathrm{rFVIIa}(10.2 \%)$ and those who received placebo $(8.7 \%)$. Considering only arterial thrombosis as outcome, the rates of thromboembolic events were higher among those who received rFVIIa than among those who received placebo (5.5 vs. $3.2 \%, p=0.003)$. Among patients who were 65 years of age or older, the risk of arterial thromboembolic events was higher for those taking rFVIIa compared to placebo group (OR 2.43, 95\% CI 1.34-4.41). The rate of venous thromboembolic events was 5.3\% among patients who received treatment, as compared with 5.7\% among patients in the placebo group with no significant statistical difference $(p=0.61)$. The rate of 
thromboembolic events in 349 healthy volunteers was the same both among those who received rFVIIa (2/232) and among who received placebo (1/117).

\section{Strength of the study}

- The use of rFVIIa on an off-label basis to treat lifethreatening bleeding has been constantly increasing, thus the evaluation of the risk of thrombotic complications is relevant.

- It is the first analysis from randomized placebocontrolled clinical trials focused only on the thromboembolic adverse events occurring in patients administrated off-label rVIIa.

\section{Weakness of the study}

- The individual study samples were relatively small, and there was clinical heterogeneity with different indications for rVIIa treatment (e.g., central nervous system bleeding, bleeding from liver disease, trauma, and other causes).

\section{Question marks}

- The statistical models used to pool the data are not clear. In the abstract, it is mentioned the use of randomeffects models while it is not described in the statistical analysis paragraph of the paper.

- The number of the total thromboembolic events reported in the abstract (498) and in the text (401) does not match.

\section{Sponsorship}

- The study was funded by Novo Nordisk, the Novo Seven producer; however, although the results were negative for the Company, the authors decided to publish the data.

\section{Clinical bottomline}

Many consider rFVIIa to be the "magic bullet" of haemostasis and believe that rFVIIa can cure all bleeding. Even without considering the effectiveness of off-label use of rVIIa, safety is a big concern. When rFVIIa is used on an off-label basis to treat life-threatening bleeding, it is mandatory to take into account the increased risk of thrombotic complications, particularly among older patients.

Conflict of interest None.

\section{References}

1. MacLaren R, Weber LA, Brake H, Gardner MA, Tanzi M (2005) A multicenter assessment of recombinant factor VIIa off-label usage: clinical experiences and associated outcomes. Transfusion. 45:1434-1442

2. Magnetti S, Oinonen M, Matuszewski KA (2007) An evaluation of off-label use of recombinant activated human factor VII (NovoSeven): patient characteristics, utilization trends, and outcomes from an electronic database of US academic health centers. Pharm Ther. 32:218-230

3. Levi M, Levy JH, Andersen HF, Truloff D (2010) Safety of Recombinant Activated Factor VII in Randomized Clinical Trials. N Engl J Med 363:1791-1800 